\title{
Longitudinal effects of physical activity on self-efficacy and cognitive processing of active and sedentary elderly women
}

\author{
Sofia Rosanti ${ }^{1}$ Guilherme Elias da Silva², Flávia Heloísa Santos ${ }^{1,3}$
}

\begin{abstract}
Previous studies support that regular physical activity in aging contributes as a protective factor against cognitive decline and improves mood states. However, there is a lack of longitudinal studies in this area. Objective: To observe possible changes in cognition related with physical activity. Methods: This study reassessed, after one-year period, 31 elderly women divided into two groups, sedentary versus active, using behavioral scales and cognitive tests. Results: The active group exhibited significantly enhanced performance in general cognitive function, particularly on tasks of episodic memory and praxis, and also on the mood states scale compared to the sedentary group. The active women also reported higher self-efficacy. Conclusion: Long-term physical activity promoted improvement on quality of life in the elderly women. Key words: aging, physical activity, self-efficacy, episodic memory, praxis.
\end{abstract}

\section{ESTUDO LONGITUDINAL SOBRE EFEITOS DA AUTOEFICÁCIA E DA PRÁTICA DE ATIVIDADES FíSICAS NO PROCESSAMENTO COGNITIVO DE IDOSAS ATIVAS E SEDENTÁRIAS}

RESUMO. Estudos anteriores sustentam que a prática regular de exercícios físicos contribui como fator de proteção aos declínios cognitivos e melhoras no estado de humor no envelhecimento. Contudo, há uma carência de estudos longitudinais nesta área. Objetivo: Observar possíveis alterações na cognição em relação a atividade física. Métodos: 0 presente estudo reavaliou após um ano 31 idosas divididas em dois grupos, sedentárias versus ativas, por meio de escalas comportamentais e testes cognitivos. Resultados: Houve um desempenho significativamente melhor em idosas ativas em funções cognitivas gerais, mais especificamente em tarefas da memória episódica e praxia, como também para a escala de estado de humor, em comparação com o grupo de sedentárias. 0 grupo de ativas também apresentou maior auto eficácia. Conclusão: Práticas de atividades físicas a longo prazo promoveu melhora na qualidade de vida de idosas.

Palavras-chave: envelhecimento, atividades físicas, auto eficácia, memória episódica, praxia.

\section{INTRODUCTION}

The elderly population has been growing 1 every year, both in developed and developing countries. ${ }^{1}$ The life expectancy of the Brazilian population increased from 63 to 73 years between 1990 and 2009, ${ }^{2}$ with a consequent higher prevalence of chronic-degenerative diseases in this population, ${ }^{3}$ particularly dementia. ${ }^{4,5}$

Biological, psychological and social changes are often observed during the aging process $^{6-9}$ and a significant portion of this population exhibits cognitive deficits, especially memory dysfunctions, ${ }^{10-12}$ affecting episodic memory more than semantic memory. ${ }^{13}$
Cognitive deficits may be strongly associated with depression. The more severe the depression, the greater the cognitive and functional impairment of patients, resulting in impaired quality of life. ${ }^{14}$ The level of education of elderly also influences the extent of decline in cognitive skills. ${ }^{15}$ In Brazil, the average schooling of elderly is 4.1 years for the country as a whole,${ }^{16}$ whereas $30.7 \%$ have less than a year of formal education. ${ }^{3}$

Mnemonic losses may be interpreted by the concept of self-efficacy, which denotes the self-evaluation an individual carries out on their own ability to perform a task within a specific field. ${ }^{17}$ Individuals with high self-

IUNESP, Universidade Estadual Paulista, Campus Bauru, SP, Brazil. UEM, Universidade Estadual de Maringá, Maringá PR, Brazil; 3Universidade do Minho, Braga, Portugal

Flávia Heloísa dos Santos. Universidade Estadual Paulista “Julio de Mesquita Filho" / UNESP / Campus Bauru. Programa de Pós-Graduação em Psicologia do Desenvolvimento e Aprendizagem - Avenida Engenheiro Luiz Edmundo Carrijo Coube, 14-01 - 17033-360 Bauru SP - Brazil. E-mail: flaviahs@psi.uminho.pt 
efficacy, who feel able to accomplish a particular task, invest greater effort to perform it, have greater motivation to complete it and persevere longer in executing the task than individuals with low self-efficacy. Therefore, perceived self-efficacy exerts a regulatory function on behavior and contributes to the quality of psychosocial functioning. ${ }^{17}$

There is a tendency toward physical inactivity among Brazilians. The percentage of people who practice physical activity regularly is $10.8 \%$ and $5.2 \%$ among men and women, respectively. ${ }^{16}$ Regular physical activity yields benefits such as higher longevity, improved cardiorespiratory and muscular capacity, aids weight control and nutrition, increases strength, and resistance in general; improves flexibility, coordination, and balance. ${ }^{18,19} \mathrm{Be}-$ sides, it promotes greater self-motivation and sense of self efficacy, ${ }^{20,21}$ preventing the development of chronic diseases and improving the quality of life in aging. ${ }^{22}$ Moreover, regular physical activity can lead to improvement of cognitive functions such as memory, attention, executive functions and praxis, ${ }^{18,23-25}$ thus constituting a protective factor for cognitive impairment in elderly. ${ }^{26-28}$

Nevertheless, there are few studies with longitudinal tracking to help ensure a better quality of life have been conducted in this population. ${ }^{29-35}$ When problems are detected early, there is a greater chance of providing adequate intervention and stable maintenance of these abilities for a longer period of time. ${ }^{36,37}$

The aims of this study were to assess possible changes in neuropsychological profile and to evaluate the concept of self-efficacy in active and sedentary elderly followed over a 12-month period by reassessing these parameters and investigating their relationship with the practice of physical activity.

\section{METHODS}

Participants were 31 elderly women, aged $60-70$ years $(\mathrm{M}=67.1, \mathrm{SD}=3.4)$ with an educational level of 4-15 years $(\mathrm{M}=6.4$ years, $\mathrm{SD}=3.3)$, living in the rural area of São Paulo State. Of the study sample, 17 were participants of the Agita Assis project - practicing physical activity at least three times a week, with each session lasting at least 50 minutes - and comprised the experimental group. The remaining 14 elderly women were sedentary - not practicing any physical activity - and comprised the control group. The women were assessed by screening and neuropsychological assessment.

\section{Instruments.}

General Screening - Anamnesis; ${ }^{26}$ Raven's Progressive Matrices Scale, series A, B, C, D and E. ${ }^{38}$ CAMCOG -
Cognitive Section of the CAMDEX; ${ }^{39,40}$ Brazil Economic Classification Criterion (CCEB); ${ }^{41}$ Geriatric Depression Scale (GDS-SF), shorter version with fifteen items; ${ }^{42}$ AUDIT (Alcohol Use Disorders Identification Test). ${ }^{43}$

Neuropsychological Assessment - Free recall of word list; ${ }^{44}$ Corsi block-tapping task; ${ }^{45}$ Phonological verbal fluency task. Letters F, A and S; ${ }^{46}$ Wechsler Adult Intelligence Scale - revised (WAIS-R) - Digit Span subtest; ${ }^{47}$ Wechsler Memory Scale third edition (WMS-III) - subtests: Logical Memory subtest; Visual Reproduction subtest; Vocabulary subtest; ${ }^{48}$ Index of Independence in Activities of Daily Living (ADL). ${ }^{49}$

Memory Self-Efficacy Questionnaire (MSEQ) ${ }^{50}$ - This is an instrument for assessing self-efficacy through seven questions based on the execution of memory tasks (for example recall of everyday objects, phone numbers, and names of people in photos) on which participants judge their own ability to achieve them. It should be noted that the memory tasks in this questionnaire mainly involve episodic memory skill. A version of the questionnaire translated and adapted for use in Brazilian samples was applied. ${ }^{51}$

Statistical analyses. For inferential purposes, repeated measures ANOVA was carried out considering group as the independent variable (sedentary versus active) and the total score obtained on each test or scale as dependent variables. Also taking into account the effects of time elapsed between the two assessment periods (baseline and after one year), differences were confirmed by the post hoc Tukey HDS test. For all comparisons, the level of significance was set at $\mathrm{p} \leq 0.05$. Effect sizes were obtained by the ETA-squared and classified as: small 0.1 , medium 0.6 and large effects $0.14 .^{52}$

Ethical aspects. The study was approved by the Research Ethics Committee of the State University of São Paulo State, "Júlio de Mesquita Filho", under case no. 464/2006.

\section{RESULTS}

The results were produced by comparing the performance achieved on tests and scales in the assessment periods by group (active and sedentary), as well as the interaction between the period and performance of each group for each variable evaluated.

The results in Table 1 showed no group effect for total score on the CAMCOG [F (1.29)<0.01, $\mathrm{p}=0.93$ ] and no effect of assessment period [ $F(1.29)=1.04, p=0.32]$. 
Table 1. Comparison between performances by groups on parameters assessed in the screening at baseline assessment and after one year.

\begin{tabular}{|c|c|c|c|c|c|c|c|c|}
\hline \multirow[b]{2}{*}{$\begin{array}{l}\text { Parameters } \\
\text { assessed }\end{array}$} & \multicolumn{2}{|c|}{ Active $(n=17)$} & \multicolumn{2}{|c|}{ Sedentary $(n=14)$} & \multirow[b]{2}{*}{ Effects } & \multirow[b]{2}{*}{$\mathbf{F}$} & \multirow[b]{2}{*}{ p } & \multirow[b]{2}{*}{$\eta 2$} \\
\hline & $\begin{array}{c}\text { Baseline } \\
\text { assessment } \\
\mathrm{M} \pm(\mathrm{SD})\end{array}$ & $\begin{array}{c}\text { After } \\
\text { one year } \\
\mathrm{M} \pm(\mathrm{SD}) \\
\end{array}$ & $\begin{array}{c}\text { Baseline } \\
\text { assessment } \\
\mathrm{M} \pm(\mathrm{SD})\end{array}$ & $\begin{array}{c}\text { After } \\
\text { one year } \\
\mathrm{M} \pm(\mathrm{SD}) \\
\end{array}$ & & & & \\
\hline \multirow{3}{*}{ CAMCOG } & \multirow{3}{*}{$87.1(8.7)$} & \multirow{3}{*}{$88.1(8.0)$} & \multirow{3}{*}{$87.6(6.8)$} & \multirow{3}{*}{$87.1(6.7)$} & Group & $<0.01$ & 0.93 & .00 \\
\hline & & & & & Period & 1.03 & 0.31 & \\
\hline & & & & & Interaction & 7.41 & $0.01^{\star}$ & \\
\hline \multirow{3}{*}{ Orientation } & \multirow{3}{*}{$9.8(0.4)$} & \multirow{3}{*}{$9.9(0.3)$} & \multirow{3}{*}{$9.7(0.5)$} & \multirow{3}{*}{$9.8(0.4)$} & Group & 0.27 & 0.60 & .02 \\
\hline & & & & & Period & 3.41 & 0.07 & \\
\hline & & & & & Interaction & 0.59 & 0.44 & \\
\hline \multirow{3}{*}{ Comprehension } & \multirow{3}{*}{$8.6(0.5)$} & \multirow{3}{*}{$8.8(0.4)$} & & & Group & 2.07 & 0.16 & .05 \\
\hline & & & $8.4(0.5)$ & $8.5(0.5)$ & Period & 2.05 & 0.10 & \\
\hline & & & & & Interaction & 0.17 & 0.68 & \\
\hline & & & & & Group & 1.20 & 0.28 & .04 \\
\hline Expression & $17.1(2.0)$ & $17.2(1.7)$ & $17.7(1.3)$ & $17.7(1.2)$ & Period & 0.07 & 0.79 & \\
\hline & & & & & Interaction & 0.07 & 0.79 & \\
\hline & & & & & Group & 0.55 & 0.46 & .01 \\
\hline $\begin{array}{l}\text { Remote } \\
\text { memory }\end{array}$ & $3.6(1.9)$ & $3.7(1.6)$ & $4.0(1.8)$ & $4.2(1.5)$ & Period & 2.42 & 0.13 & \\
\hline & & & & & Interaction & 0.20 & 0.65 & \\
\hline & & & & & Group & 1.31 & 0.26 & .06 \\
\hline $\begin{array}{l}\text { Recent } \\
\text { memory }\end{array}$ & $3.4(0.8)$ & $3.4(0.6)$ & $3.7(0.6)$ & $3.6(0.6)$ & Period & 0.23 & 0.63 & \\
\hline & & & & & Interaction & 1.36 & 0.25 & \\
\hline & & & & & Group & 0.12 & 0.72 & .01 \\
\hline $\begin{array}{l}\text { Learning } \\
\text { memory }\end{array}$ & $13.5(1.7)$ & $14.3(1.2)$ & $13.8(2.0)$ & $13.6(1.6)$ & Period & 4.91 & $0.03^{*}$ & \\
\hline & & & & & Interaction & 14.25 & $<0.01^{*}$ & \\
\hline & & & & & Group & 0.58 & 0.45 & .03 \\
\hline Attention & $4.6(1.8)$ & $4.6(1.5)$ & $5.2(1.8)$ & $4.9(1.5)$ & Period & 0.97 & 0.33 & \\
\hline & & & & & Interaction & 2.24 & 0.14 & \\
\hline & & & & & Group & 25.05 & $<0.01^{*}$ & $.49^{\#}$ \\
\hline Praxis & $9.9(0.8)$ & $9.9(0.8)$ & $8.3(0.9)$ & $8.5(0.9)$ & Period & 1.46 & 0.23 & \\
\hline & & & & & Interaction & 3.38 & 0.07 & \\
\hline & & & & & Group & 0.01 & 0.89 & .00 \\
\hline Math & $1.9(0.2)$ & $1.9(0.2)$ & $1.9(0.3)$ & $1.9(0.3)$ & Period & 428.30 & $<0.01^{*}$ & \\
\hline & & & & & Interaction & 0.00 & 1.00 & \\
\hline & & & & & Group & 0.19 & 0.66 & .00 \\
\hline $\begin{array}{l}\text { Abstract } \\
\text { thinking }\end{array}$ & $5.5(1.8)$ & $5.3(1.6)$ & $5.5(1.1)$ & $5.7(1.0)$ & Period & 0.04 & 0.83 & \\
\hline & & & & & Interaction & 5.07 & $0.03^{*}$ & \\
\hline & & & & & Group & 2.82 & 0.10 & .09 \\
\hline Perception & $9.1(1.4)$ & $9.0(1.4)$ & $8.4(0.7)$ & $8.3(1.0)$ & Period & 0.38 & 0.54 & \\
\hline & & & & & Interaction & $<0.01$ & 0.95 & \\
\hline & & & & & Group & 0.59 & 0.44 & .02 \\
\hline ABEP & $15.2(5.2)$ & $15.8(4.6)$ & $16.3(4.1)$ & $17.0(3.9)$ & Period & 8.40 & $<0.01^{\star}$ & \\
\hline & & & & & Interaction & 0.07 & 0.78 & \\
\hline & & & & & Group & 0.95 & 0.34 & .02 \\
\hline $\begin{array}{l}\text { Raven } \\
\text { test }\end{array}$ & $37.7(8.0)$ & $38.6(7.4)$ & $35.8(5.2)$ & $35.8(4.8)$ & Period & 3.55 & 0.69 & \\
\hline & & & & & Interaction & 3.55 & 0.69 & \\
\hline & & & & & Group & 0.41 & 0.53 & .01 \\
\hline AUDIT & $0.1(0.5)$ & $0.1(0.5)$ & $0.3(1.0)$ & $0.4(1.3)$ & Period & 1.22 & 0.27 & \\
\hline & & & & & Interaction & 1.22 & 0.27 & \\
\hline & & & & & Group & 0.90 & 0.34 & .00 \\
\hline GDS-SF & $2.2(1.6)$ & $2.0(1.6)$ & $2.2(1.7)$ & $3.2(2.1)$ & Period & 2.23 & 0.14 & \\
\hline & & & & & Interaction & 7.52 & $0.01^{*}$ & \\
\hline
\end{tabular}

M: mean; SD: standard deviation; ABEP: Scale for assessment of Brazilian economic classification; Raven test: Raven's Progressive Matrices; AUDIT: Alcohol Use Disorders Identification Test; GDS-SF: Geriatric Depression Scale, short form (*) $p<0.05$; (\#) Eta-squared large effect: $\eta 2>0.14$. 
Table 2. Comparisons between performances by Groups on parameters assessed by neuropsychological assessment at baseline and after one year.

\begin{tabular}{|c|c|c|c|c|c|c|c|c|}
\hline \multirow[b]{2}{*}{$\begin{array}{l}\text { Parameters } \\
\text { assessed }\end{array}$} & \multicolumn{2}{|c|}{ Active $(\mathrm{n}=17)$} & \multicolumn{2}{|c|}{ Sedentary $(n=14)$} & \multirow[b]{2}{*}{ Effects } & \multirow[b]{2}{*}{$\mathbf{F}$} & \multirow[b]{2}{*}{$\mathbf{p}$} & \multirow[b]{2}{*}{$\eta 2$} \\
\hline & $\begin{array}{c}\text { Baseline } \\
\text { assessment } \\
\mathrm{M} \pm(\mathrm{SD})\end{array}$ & $\begin{array}{c}\text { After } \\
\text { one year } \\
M \pm(S D)\end{array}$ & $\begin{array}{c}\text { Baseline } \\
\text { assessment } \\
\mathrm{M} \pm(\mathrm{SD})\end{array}$ & $\begin{array}{c}\text { After } \\
\text { one year } \\
\text { M } \pm(S D)\end{array}$ & & & & \\
\hline \multirow[t]{3}{*}{ MSEQ } & $52.9(11.9)$ & $54.4(10.9)$ & $48.6(12.3)$ & $46.8(10.7)$ & Group & 2.14 & 0.15 & 0.03 \\
\hline & & & & & Period & 0.02 & 0.88 & \\
\hline & & & & & Interaction & 7.07 & $0.01^{*}$ & \\
\hline \multirow{3}{*}{$\begin{array}{l}\text { Free word recall } \\
\text { immediate }\end{array}$} & $5.9(2.0)$ & $5.4(1.6)$ & $4.2(1.4)$ & $5.4(21.5)$ & Group & 3.46 & 0.07 & $0.20^{\#}$ \\
\hline & & & & & Period & 0.04 & 0.83 & \\
\hline & & & & & Interaction & 1.92 & 0.18 & \\
\hline \multirow{3}{*}{$\begin{array}{l}\text { Free word recall } \\
\text { delayed }\end{array}$} & $3.1(2.0)$ & $3.5(1.3)$ & $2.0(1.2)$ & $2.7(1.4)$ & Group & 6.45 & $0.01^{*}$ & 0.09 \\
\hline & & & & & Period & 0.55 & 0.46 & \\
\hline & & & & & Interaction & 9.25 & $<0.01^{*}$ & \\
\hline \multirow{3}{*}{$\begin{array}{l}\text { Visual Reproduction } \\
\text { Immediate - A }\end{array}$} & $4.8(1.5)$ & $5.2(1.1)$ & $4.6(0.9)$ & $4.1(1.3)$ & Group & 2.25 & 0.14 & 0.01 \\
\hline & & & & & $4.8(1.5)$ & 0.55 & 0.46 & \\
\hline & & & & & Interaction & 9.85 & $<0.01^{\star}$ & \\
\hline \multirow{3}{*}{$\begin{array}{l}\text { Visual Reproduction } \\
\text { Immediate - B }\end{array}$} & $5.0(1.9)$ & $5.2(1.5)$ & $3.8(1.8)$ & $3.8(1.7)$ & Group & 4.23 & $0.04^{*}$ & 0.09 \\
\hline & & & & & Period & 0.33 & 0.56 & \\
\hline & & & & & Interaction & 1.84 & 0.18 & \\
\hline \multirow{3}{*}{$\begin{array}{l}\text { Visual Reproduction } \\
\text { Immediate - C }\end{array}$} & $7.5(1.8)$ & $7.3(1.7)$ & $6.1(2.0)$ & $5.7(1.9)$ & Group & 5.29 & $0.02^{*}$ & 0.12 \\
\hline & & & & & Period & 5.30 & $0.02^{*}$ & \\
\hline & & & & & Interaction & 0.44 & 0.51 & \\
\hline \multirow{3}{*}{$\begin{array}{l}\text { Visual Reproduction } \\
\text { Immediate - D }\end{array}$} & $13.6(2.3)$ & $13.8(2.2)$ & $12.7(3.5)$ & $12.1(3.0)$ & Group & 1.63 & 0.21 & 0.02 \\
\hline & & & & & Period & 1.74 & 0.19 & \\
\hline & & & & & Interaction & 6.25 & $0.01^{*}$ & \\
\hline \multirow{3}{*}{$\begin{array}{l}\text { Visual reproduction } \\
\text { Delayed-A }\end{array}$} & $4.1(1.9)$ & $4.6(1.2)$ & $3.6(1.9)$ & $3.1(1.5)$ & Group & 3.09 & 0.08 & 0.02 \\
\hline & & & & & Period & 0.04 & 0.82 & \\
\hline & & & & & Interaction & 4.28 & $0.04^{*}$ & \\
\hline \multirow{3}{*}{$\begin{array}{l}\text { Visual reproduction } \\
\text { Delayed-B }\end{array}$} & $4.4(2.1)$ & $4.7(1.7)$ & $3.2(2.0)$ & $2.7(1.7)$ & Group & 5.42 & $0.02^{\star}$ & 0.07 \\
\hline & & & & & Period & 0.23 & 0.63 & \\
\hline & & & & & Interaction & 3.42 & 0.07 & \\
\hline \multirow{3}{*}{$\begin{array}{l}\text { Visual reproduction } \\
\text { Delayed-C }\end{array}$} & $6.9(1.8)$ & $6.2(1.9)$ & $5.9(1.8)$ & $5.2(1.7)$ & Group & 2.83 & 0.10 & 0.09 \\
\hline & & & & & Period & 8.77 & $<0.01^{*}$ & \\
\hline & & & & & Interaction & 0.06 & 0.79 & \\
\hline \multirow{3}{*}{$\begin{array}{l}\text { Visual reproduction } \\
\text { Delayed-D }\end{array}$} & $11.6(3.4)$ & $11.5(2.9)$ & $10.8(3.6)$ & $10.0(2.9)$ & Group & 0.98 & 0.32 & 0.01 \\
\hline & & & & & Period & 2.38 & 0.13 & \\
\hline & & & & & Interaction & 1.30 & 0.26 & \\
\hline \multirow{3}{*}{$\begin{array}{l}\text { Logical Memory } \\
\text { Immediate }\end{array}$} & $12.9(3.1)$ & $11.6(2.3)$ & $11.6(2.9)$ & $11.1(2.5)$ & Group & 3.50 & 0.07 & 0.05 \\
\hline & & & & & Period & 0.24 & 0.62 & \\
\hline & & & & & Interaction & 6.22 & $0.02^{\star}$ & \\
\hline \multirow{3}{*}{$\begin{array}{l}\text { Logical Memory } \\
\text { Delayed }\end{array}$} & $11.2(3.5)$ & $10.0(2.5)$ & $9.1(2.6)$ & 8.7 (2.3) & Group & 4.94 & $0.03^{*}$ & 0.10 \\
\hline & & & & & Period & 1.27 & 0.26 & \\
\hline & & & & & Interaction & 1.27 & 0.26 & \\
\hline Corsi Blocks: & $6.8(1.3)$ & $6.7(1.4)$ & $6.8(1.2)$ & $6.6(0.9)$ & Group & 0.01 & 0.91 & 0.00 \\
\hline Forward & & & & & Period & 1.67 & 0.21 & \\
\hline & & & & & Interaction & 1.67 & 0.21 & \\
\hline Corsi Blocks: & $5.0(1.6)$ & $5.1(1.5)$ & $5.5(1.8)$ & $5.1(1.4)$ & Group & 0.21 & 0.65 & 0.02 \\
\hline Backward & & & & & Period & 1.85 & 0.18 & \\
\hline & & & & & Interaction & 3.23 & 0.08 & \\
\hline Digits - forward & $7.1(1.1)$ & $7.2(1.1)$ & $6.5(1.2)$ & $6.4(1.2)$ & Group & 2.60 & 0.11 & 0.06 \\
\hline order & & & & & Period & 0.07 & 0.79 & \\
\hline & & & & & Interaction & 1.18 & 0.28 & \\
\hline
\end{tabular}


Table 2. Continuation.

\begin{tabular}{|c|c|c|c|c|c|c|c|c|}
\hline \multirow[b]{2}{*}{$\begin{array}{l}\text { Parameters } \\
\text { assessed }\end{array}$} & \multicolumn{2}{|c|}{ Active $(\mathrm{n}=17)$} & \multicolumn{2}{|c|}{ Sedentary $(n=14)$} & \multirow[b]{2}{*}{ Effects } & \multirow[b]{2}{*}{$\mathbf{F}$} & \multirow[b]{2}{*}{$\mathbf{p}$} & \multirow[b]{2}{*}{$\eta 2$} \\
\hline & $\begin{array}{c}\text { Baseline } \\
\text { assessment } \\
\mathrm{M} \pm(\mathrm{SD})\end{array}$ & $\begin{array}{c}\text { After } \\
\text { one year } \\
\mathrm{M} \pm(\mathrm{SD})\end{array}$ & $\begin{array}{c}\text { Baseline } \\
\text { assessment } \\
\mathrm{M} \pm(\mathrm{SD})\end{array}$ & $\begin{array}{c}\text { After } \\
\text { one year } \\
M \pm(S D)\end{array}$ & & & & \\
\hline \multirow{3}{*}{$\begin{array}{l}\text { Digits - backward } \\
\text { order }\end{array}$} & $4.6(1.0)$ & $4.8(0.9)$ & $4.4(1.3)$ & $4.3(1.4)$ & Group & 0.61 & 0.44 & 0.00 \\
\hline & & & & & Period & 0.04 & 0.84 & \\
\hline & & & & & Interaction & 3.67 & 0.06 & \\
\hline \multirow[t]{3}{*}{ Fluency - F } & $10.1(3.0)$ & $10.2(2.6)$ & $10.9(3.5)$ & $10.9(3.3)$ & Group & 0.45 & 0.50 & 0.02 \\
\hline & & & & & Period & $<0.01$ & 0.96 & \\
\hline & & & & & Interaction & 0.27 & 0.60 & \\
\hline \multirow[t]{3}{*}{ Fluency - A } & $8.5(2.1)$ & $8.8(1.9)$ & $9.4(2.8)$ & $9.4(2.7)$ & Group & 0.79 & 0.38 & 0.03 \\
\hline & & & & & Period & 0.47 & 0.49 & \\
\hline & & & & & Interaction & 0.47 & 0.49 & \\
\hline \multirow[t]{3}{*}{ Fluency-S } & $8.4(2.0)$ & $8.6(1.7)$ & $8.9(3.0)$ & $8.7(2.9)$ & Group & 0.14 & 0.71 & 0.01 \\
\hline & & & & & Period & 0.11 & 0.73 & \\
\hline & & & & & Interaction & 1.89 & 0.17 & \\
\hline \multirow[t]{3}{*}{ Vocabulary } & $31.9(5.2)$ & $32.4(4.7)$ & $33.1(6.2)$ & $32.5(5.7)$ & Group & 0.10 & 0.74 & 0.01 \\
\hline & & & & & Period & 0.34 & 0.56 & \\
\hline & & & & & Interaction & 5.44 & $0.02^{\star}$ & \\
\hline \multirow[t]{3}{*}{$\mathrm{ADL}$} & $2.29(1.0)$ & $2.29(1.2)$ & $3.7(1.6)$ & $4.7(1.7)$ & Group & 17.14 & $<0.01^{*}$ & $0.24^{\#}$ \\
\hline & & & & & Period & 8.56 & $<0.01^{*}$ & \\
\hline & & & & & Interaction & 8.56 & $<0.01^{*}$ & \\
\hline
\end{tabular}

M: mean, SD: standard deviation; MSEQ: Memory Self-Efficacy Questionnaire; ADL: Index of Independence in Activities of Daily Living. ( $\left(^{\star}\right) p<0.05$; (\#) Eta-squared large effect: $\eta 2>0.14$.

However, a significant interaction was observed [F (1.29) $=7.41, \mathrm{p}=0.01]$.

There was no significant difference between groups across the CAMCOG subtests assessing episodic memory [F (1.29) $=0.13, \mathrm{p}=0.72]$, however, an effect for assessment period was noted $[\mathrm{F}(1.29)=4.91, \mathrm{p}=0.03$ ], i.e., a better performance by all participants was evident at the second assessment compared to the first. Additionally, there was an interaction effect between group and assessment period $[\mathrm{F}(1.29)=14.2, \mathrm{p}<0.01]$.

A significant difference between the groups was found for praxis skills $[F(1.29)=25.05, p<0.01]$ at both assessment periods, in that the active group performed better than the sedentary group. There was no significant difference in socioeconomic status between the groups. However, an effect for assessment period [ $\mathrm{F}(1.29)=8.40$, $\mathrm{p}<0.01]$ was detected by the post hoc Tukey test, i.e., an increase in the average economic level of all participants at the second assessment according to $\mathrm{ABEP}{ }^{41}$

No group [ $\mathrm{F}(1.29)=0.91, \mathrm{p}=0.35]$ or assessment period effects $[F(1.29)=2.24, p=0.14]$ were found on the Geriatric Depression Scale though an interaction among these items $[\mathrm{F}(1.29)=7.53, \mathrm{p}=0.01]$ was detected. Although the sedentary group, after one year, had higher scores on GDS-SF, the overall mean score of the group was less than 5 (cut off indicating depression). Notably, a year after the baseline assessment, one subject attained a score of over 5 on the GDS-SF (cut off recommended by the scale) in this group, i.e. symptoms indicative of mild depression.

There was a difference in self-report by the elderly for both groups regarding their ability to perform tasks involving memory usage, since the sedentary elderly had a lower sense of self-efficacy in relation to the active group. Results for the active group at baseline assessment were: Capacity (decrease 23.5\%; maintained 58.8\%; increase $17.7 \%$ ), whereas after one year (decrease $23.5 \%$; maintained $52.3 \%$; increase $23.6 \%$ ). The sedentary group at baseline assessment: Capacity (decrease $50.0 \%$; maintain $42.8 \%$; increase $7.2 \%$ ) whereas after one year (decrease $57.1 \%$; maintain $35.7 \%$; increase $7.2 \%$ ).

The total score difference in performance on the Memory Self-Efficacy Questionnaire (MSEQ) was not significant $[\mathrm{F}(1.29)=2.14, \mathrm{p}=0.15]$ and there was no assessment period effect $[F(1.29)=0.02, p=0.88]$. How ever, there was a significant interaction $[\mathrm{F}(1.29)=7.07$, $\mathrm{p}=0.01$ ] in self-assessment of ability to perform tasks involving memory usage, especially long-term episodic memory, after one year.

On the longitudinal neuropsychological assessment applied one year after the baseline assessment - a significant improvement was observed in the active group performance compared to the sedentary group on Free Words Recall, Visual Reproduction and Logical Memory tasks assessing episodic memory subtype performance and also on the scale for assessing activities of daily living (Table 2). 


\section{DISCUSSION}

The aim of the study was to determine possible changes in neuropsychological profile and the concept of selfefficacy of active and sedentary elderly women after 12 months by reassessing these parameters to investigate their relationship with physical activity.

For total score on the CAMCOG general cognitive screening test, there were no group or assessment-period effects. ${ }^{40}$ However, the significant interaction indicates an increase in scores over time for the active group and a slight decrease in scores for the sedentary group after one year. This interaction suggests that over the year, the general cognitive performance was better in the group of elderly practicing regular physical activity, ${ }^{23-25}$ showing that participation in physical activity programs benefits the physical and psychological spheres and that physically active individuals most likely have faster cognitive processing. ${ }^{18,19}$ This suggests that physical activity may be an important protective factor against cognitive impairment and dementia in elderly. ${ }^{26,27}$

However, the scores on subtests of the CAMCOG for episodic memory tasks indicate improvement in performance on learning memory (Table 1) by all participants in the active elderly group after one year compared to baseline assessment. Physical activity might have positively affected the scores, but differences were not detected by Tukey's post hoc test. These were confirmed however, on the neuropsychological tests, one year after the baseline assessment by specific tests for episodic memory, mainly in delayed recall (Table 2). The active group also had a higher degree of self-efficacy for the tasks involving higher memory capacity. ${ }^{13,17}$ This provides indirect evidence that regular practice of physical activity is associated with an improvement in motivation and sense of self-efficacy. ${ }^{20,21}$

In the evaluation of praxis by the CAMCOG, the active group performed better than the sedentary group at both assessment periods (baseline and after one year). There was a positive association with the scores obtained on the Index of Independence in Activities of Daily Living, in which the active elderly performed significantly better than the sedentary group. This association indicates that the limitations in praxis of the sedentary group interfere in daily living. ${ }^{18,19,23-25}$

Despite significant differences between the active and sedentary elderly on tasks involving episodic memory skills and praxis, there were no group differences for vocabulary, phonological and semantic verbal fluency or working memory (Table 1), consistent with the notion that episodic memory decline prevails over semantic memory impairment in the non-pathological aging process. ${ }^{13}$
The Memory Self-Efficacy Questionnaire (MSEQ) revealed an interaction between performance by group and assessment period, showing an increase in scores after one year for the active group and a slight decrease in scores for the sedentary group in the self-assessment of ability to perform tasks involving memory usage. Considering that individuals' perceptions of their efficacy affect the projections and predictions about the outcome of their actions, it follows that a negative evaluation of self-efficacy for memory predicts failure while a positive evaluation predicts success. ${ }^{17}$

With regards to mood states, no difference in the performance of each group for scores on the GDS-SF was evident, as there was no assessment period effect. Nevertheless, there was a significant interaction, which indicates an increase in scores after one year in the sedentary group and a slight decrease in scores for the active group, indicating greater depressive symptoms in the former group and fewer in the latter after one year. Thus, these results suggest that the mood of active elderly was significantly better than that reported by the sedentary women.

Although some cognitive functions are negatively affected by age due to the loss of neurons concomitant with decline in cognitive performance, ${ }^{6}$ the processes based on crystallized abilities, such as verbal knowledge and comprehension, remain unaffected or improve with aging. On the other hand, procedures based on fluid abilities, such as learned tasks but not implemented tasks, may suffer decline. ${ }^{7}$ This fact is evidenced by the results obtained in our study, as significant differences were found, revealing better performance of active elderly compared to sedentary elderly on activities involving skills, episodic memory and praxis, but not tasks of vocabulary, verbal, semantic and phonological fluency or working memory.

Regarding limitations of this study, the low number of participants should be considered. Convenience sampling was used, however, the ETA-squared index showed medium and large effect sizes for most of the dependent variables. Another issue of concern may be the time elapsed between baseline and follow-up assessments. However, based on previous studies we may assume that a one-year follow-up period is a reliable interval. ${ }^{12,28}$ In addition, we detected cognitive changes in this short timeframe, which may serve as valuable information for rehabilitation purposes.

In summary, active elderly exhibited superior performance compared to the sedentary group with regards to episodic memory, praxis skills and mood state. The practice of regular physical activity accompanied a 
greater sense of self-efficacy. Therefore, the results of this longitudinal study suggest that regular physical activity may be an important protective factor against cognitive impairment, dementia and depression in the elderly, ${ }^{26,27}$ and serve as an early intervention in order to reduce these effects. ${ }^{14,36,37}$

\section{REFERENCES}

1. Brucki SMD. Epidemiology of Mild Cognitive Impairment in Brazil. Dement Neuropsychol 2013;7:363-366.

2. Instituto Brasileiro de Geografia e Estatística (IBGE). Síntese de Indicadores Sociais: Uma análise das condições de vida da população brasileira, 2010.

3. Caramelli P, Barbosa MT, Sakurai E, et al. The Pietà study: Epidemiological Investigation on successful brain aging in Caeté (MG), Brazil, methods and baseline cohort characteristics. Arq Neuropsiquiatr 2011; 69:579-584.

4. Classificação Estatística Internacional de Doenças e Problemas Relacionados à Saúde (CID-10). Organização Mundial de Saúde; 2010.

5. Diagnostic and Statistical Manual of Mental Disorders, fifth edition, (DSM-5). American Psychiatric Association; 2013.

6. Colcombe SJ, Erickson KI, Raz N, et al. Aerobic fitness reduces brain tissue loss in aging humans. J Gerontol A Biol Sci Med Sci 2003;58: 176-180.

7. Kramer AF, Willis SL. Enhancing the cognitive vitality of older adults. Curr Direc Psychol Science 2002:11:173-177.

8. Drachman DA. Aging and the brain: a new frontier. Ann Neurol 1997;42: 819-828.

9. Foster TC. Biological markers of age-related memory deficits: treatment of senescent physiology. CNS Drugs 2006;20:153-166.

10. Carvalho FCR, Neri AL, Yassuda MS. Treino da memória episódica com ênfase em categorização em idosos sem demência e depressão. Psicol Reflex Crit 2010;23:317-323

11. Bertolucci PHF, Okamoto IH, Brucki SMD, Siviero MO, Neto JT, Ramos LR. Applycability of the CERAD Neuropsychological Battery to Brazilian elderly. Arq Neuropsiquiatr 2001;59:532-536.

12. van de Kommer T, Comjs HC, Rijs KJ, Heyman, MW, van Boxtel MPJ, Deeg DJH. Classificantion models for identification of at-risk groups for incident memory complaints. Int Psychogeriatr 2014;26:257-271.

13. Yassuda MS, Lasca VB, Neri AL. Meta-memória e auto-eficácia: um estudo de validação de instrumentos de pesquisa sobre memória e envelhecimento. Psicol Reflex Crít 2005;18:79-80.

14. Ávila R, Bottino CMC. Atualização Sobre Alterações Cognitivas em Idosos com Síndrome Depressiva. Rev Bras Psiq 2006:28:316-320.

15. Brucki SMD. Illiteracy and Dementia. Dement Neuropsychol 2010;4: 153-157.

16. Instituto Brasileiro de Geografia e Estatística (IBGE). Síntese de Indicadores Sociais: Uma análise das condições de vida da população brasileira; 2009.

17. Bandura A. Self-efficacy: The exercise of control. New York: W.H. Freeman; 1997.

18. Antunes HKM, Heredia RA, Bueno OF. Alterações Cognitivas em Idosas Decorrentes do Exercício Físico Sistematizado. Rev Sobama 2001; 6:27-33.

19. Wilmore $J H$, Costil D. Fisiologia do esporte e do exercício. São Paulo: Manole; 2001.

20. Costa GA. Tempo de ser: atividade física, qualidade de vida, envelhecimento e a trama das interações sociais interferindo na relação de gênero. Rev Sobama 2001;6:9-18.

21. Leão-Junior R. Participação em hidroginástica, crenças de auto eficácia e satisfação com a vida em mulheres de 50 a 70 anos. Dissertação de mestrado em Gerontologia, Faculdade de Educação da UNICAMP, Campinas, 2003.

22. Borges MRD, Moreira AK. Influências da prática de atividades físicas na terceira idade: estudo comparativo dos níveis de autonomia para o desempenho das ADVs e AIVDSs entre idosos ativos fisicamente e idosos sedentários. Motriz, Rio Claro 2009;15:562-573.

23. de Mello MT, Boscolo RA, Esteves AM, Tufik S. O exercício físico e os aspectos psicobiológicos. Rev Bras Med Esporte 2005;11:203-207.

24. Lopes KMDC. Os efeitos crônicos do exercício físico aeróbio nos níveis de serotonina e depressão em mulheres com idade entre 50 e 72 anos. Dissertação Mestrado. Brasília: Universidade Católica de Brasília; 2001.

25. Willians $\mathrm{P}$, Lord SR. Effects of group exercise on cognitive functioning and mood in older women. Aust N Z J Public Health 1997;21:45-52.

26. Silva GE, Santos FH. Efeito do sedentarismo nas funções cognitivas de idosas com escolaridade intermediária. PSICO 2009;40:81-87.

27. Soares RM, Diniz AB, Cattuzzo MT. Associação entre atividade física, aptidão física e desempenho cognitivo em idosos. Motricidade 2013; 9:84-93.
28. Alosco ML, Spitznagel MB, Cohen R, et al. Decreased physical activity predicts cognitive dysfunction and reduced cerebral blood flow in heart failure. J Neurol Sci 2014;339:169-175.

29. Argimon IIL, Stein LM. Habilidades cognitivas em indivíduos muito idosos: um estudo longitudinal. Cad Saúde Pública 2005;21:64-72.

30. Brucki SMD. Envelhecimento e memória. In: Andrade, VM, Santos, FH, Bueno, OFA. (orgs.) Neuropsicologia Hoje. São Paulo: Artes Médicas; 2004.

31. Hototian SR, Lopes MA, Azevedo D, et al. Prevalence of cognitive and functional impairment in a community sample from São Paulo, Brazil. Dement Geriatr Cogn Disord 2008;25:135-143.

32. Tamai SAB, Paschoal, SMP, Litvoc J, et al. Impact of a program to promote health and quality of life of elderly. Einstein 2011;9:8-13.

33. Veras RP. Modelos contemporâneos no cuidado à saúde: novos desafios em decorrência da mudança do perfil epidemiológico da população brasileira. Revista USP 2001;51:72-85.

34. Veras, RP, Lourenço, R, Martins, CSF, Sanches, MA, Chaves, PH. Novos paradigmas do modelo assistencial no setor de saúde: conseqüência da explosão populacional dos idosos no Brasil. In: Veras, RP (Ed.) Terceira idade: gestão contemporânea em saúde. Rio de Janeiro: Ed. Relume-Dumará/UnATI; 2002.

35. Zimerman Gl. Velhice: aspectos biopsicossociais. Porto Alegre: Artmed; 2000.

36. Fichman-Charchat H, Caramelli P, Sameshima K, Nitrini R. Declínio da Capacidade Cognitiva durante o envelhecimento. Rev Bras Psiquiatr 2005;27:79-82

37. Hamdan AC, Bueno OFA. Relações entre controle executivo e memória episódica verbal no comprometimento cognitivo leve e na demência tipo Alzheimer. Est Psicologia 2005;10:63-71.

38. Raven JC. Teste das Matrizes Progressivas - Escala Geral (Séries A, B, C, D e E). Rio de Janeiro: CEPA; 2003.

39. Huppert FA, Mountjov CQ, Tym E, Goddard R, Miller N, Roth M. Assesment of cognitive function and the diagnosis of dementia by CAMDEX. Cambridge, New York, New Rochelle, Melboune, Sidney: Cambridge University Press; 1988.

40. Bottino CMC, Almeida OP, Tamai S, Forlenza OV, Scalco MZ, Carvalho IAM. Entrevista estruturada para diagnóstico de transtornos mentais em idosos. São Paulo: PROTER; 1999.

41. Associação Brasileira de Empresas de Pesquisa (ABEP). Critério de Classificação Econômica Brasil; 2003 (http://www.abep.org).

42. Yesavage JA, Brink TL, Rose TL, et al. Development and validation of a geriatric depression screening scale: a preliminary report. J Psychiat Res 1983;17:37-49.

43. Saunders JB, Aasland OG, Babor TF, de la Fuente JR, Grant M. Development of the Alcohol Use Disorders Identification Test (AUDIT): WHO Collaborative Project on Early Detection of Persons with Harmful Alcohol Consumption-II. Addiction 1993;88:791-804.

44. Bueno OFA. Incremento de recordação livre por relacionamento semântico entre palavras: processamento automático ou que demanda atenção? Tese de Doutorado. São Paulo: Universidade Federal de São Paulo, 2001.

45. Kessels RPC, van Zandvoort MJE, Postma A, Kappelle LJ, de Haan EHF. The Corsi Block-tapping task: standardization and Normative data. Appl Neuropsychol 2000;7:252-258.

46. Lezak MD. Neuropsychological Assessment. New York: Oxford University Press; 1995

47. Wechsler D. Wechsler Adult Intelligence Scale - revised. Nova York: Psychological Corporation; 1981.

48. Wechsler D. Wechsler memory scale - third edition. San Antonio, TX: The Psychological Corporation; 1997.

49. Katz S, Ford AB, Moskowitz RW, Jackson BA, Jaffe MW. Studies of illness in the aged. The index of adl: a standardized measure of biological and psychosocial function. JAMA 1963;185:914-919.

50. Berry JM, West RL, Dennhey DM. Reliability and validity of the memory self-efficacy questionnaire. Dev Psychology 1989;25:701-713.

51. Yassuda MS, Lasca VB, Neri AL. Meta-memória e auto-eficácia: um estudo de validação de instrumentos de pesquisa sobre memória e envelhecimento. Psicol Refl Crít 2005:18:78-90.

52. Murphy KR, Myors B. Statistical power analysis: A simple and General Model for Traditional and Modern Hypothesis. (2nd ed.). London: Lawrence Erlbaum; 2004:172. 